\title{
BMJ Open Longitudinal assessment of brain- derived neurotrophic factor in Sardinian psychotic patients (LABSP): a protocol for a prospective observational study
}

\author{
Diego Primavera, ${ }^{1}$ Mirko Manchia, ${ }^{1,2}$ Luca Deriu, ${ }^{1}$ Massimo Tusconi, ${ }^{1}$ \\ Roberto Collu, ${ }^{3}$ Maria Scherma, ${ }^{3}$ Paola Fadda, ${ }^{3,4}$ Walter Fratta, ${ }^{3,4}$ \\ Bernardo Carpiniello ${ }^{1}$
}

To cite: Primavera D, Manchia M, Deriu L, et al. Longitudinal assessment of brain-derived neurotrophic factor in Sardinian psychotic patients (LABSP): a protocol for a prospective observational study. BMJ Open 2017;7:e014938. doi:10.1136/ bmjopen-2016-014938

- Prepublication history for this paper is available online. To view these files please visit the journal online (http://dx.doi org/10.1136/bmjopen-2016014938).

Received 27 0ctober 2016 Revised 20 March 2017 Accepted 31 March 2017

CrossMark

${ }^{1}$ Section of Psychiatry, Department of Medical Science and Public Health, University of Cagliari, Cagliari, Italy

${ }^{2}$ Department of Pharmacology, Dalhousie University, Halifax,

Canada

${ }^{3}$ Department of Biomedical

Sciences, Division of

Neuroscience and Clinical

Pharmacology, University of

Cagliari, Cagliari, Italy

${ }^{4}$ Centre of Excellence

'Neurobiology of Dependence', University of Cagliari, Cagliari, Italy

Correspondence to

Prof Bernardo Carpiniello;

bcarpini@iol.it

\section{ABSTRACT}

Introduction Brain-derived neurotrophic factor (BDNF) plays a crucial role in neurodevelopment, synaptic plasticity and neuronal function and survival. Serum and plasma BDNF levels are moderately, but consistently, decreased in patients with schizophrenia (SCZ) compared with healthy controls. There is a lack of knowledge, however, on the temporal manifestation of this decline. Clinical, illness course and treatment factors might influence the variation of BDNF serum levels in patients with psychosis. In this context, we propose a longitudinal study of a cohort of SCZ and schizophrenic and schizoaffective disorder (SAD) Sardinian patients with the aim of disentangling the relationship between peripheral BDNF serum levels and changes of psychopathology, cognition and drug treatments. Methods and analysis Longitudinal assessment of BDNF in Sardinian psychotic patients (LABSP) is a 24-month observational prospective cohort study. Patients with SAD will be recruited at the Psychiatry Research Unit of the Department of Medical Science and Public Health, University of Cagliari and University of Cagliari Health Agency, Cagliari, Italy. We will collect BDNF serum levels as well as sociodemographic, psychopathological and neurocognitive measures. Structured, semistructured and self-rating assessment tools, such as the Positive and Negative Syndrome Scale for psychopathological measures and the Brief Assessment of Cognition in Schizophrenia for cognitive function, will be used.

Ethics and dissemination This study protocol was approved by the University of Cagliari Health Agency Ethics Committee (NP2016/5491). The study will be conducted in accordance with the principles of good clinical practice, in the Declaration of Helsinki in compliance with the regulations. Participation will be voluntary and written informed consent will be obtained for each participant upon entry into the study. We plan to disseminate the results of our study through conference presentations and publication in international peer-reviewed journals. Access to raw data will be available in anonymised form upon request to the corresponding author.

\section{Strengths and limitations of this study}

- This is a prospective cohort study of patients with psychosis aiming to clarify whether longitudinal changes in brain-derived neurotrophic factor (BDNF) serum levels are correlated with psychopathological, cognitive, and treatment factors.

- The secondary outcomes of this study will offer insight on the impact of psychiatric comorbidities on longitudinal trajectory of BDNF in patients with psychosis.

- Sampling will be performed in a tertiary clinic specialised in treatment of patients with psychosis, possibly limiting the ecological validity of the study results.

- The sample size, based on previous work on longitudinal change of BDNF over time, might not achieve sufficient statistical power to detect association signals with small effect sizes between clinical predictors and BDNF levels.

- Notwithstanding the prospective design, clinical (illness duration, duration of untreated psychosis) and treatment history prior to study entry might impact on findings.

\section{INTRODUCTION}

Brain-derived neurotrophic factor (BDNF) is the most widely distributed neurotrophin in the central nervous system (CNS), ${ }^{1}$ being expressed in almost all the cortical areas, as well as in several spinal cord regions. ${ }^{2}$ There is substantial evidence that BDNF plays a crucial role during brain development, as well as in the process of differentiation, synaptogenesis and neuronal plasticity. ${ }^{3-6}$ BDNF was the second neurotrophic factor to be characterised after the nerve growth factor. ${ }^{7}$ Similar to other neurotrophins, BDNF is synthesised in a pre-pro form consisting of 47 amino acids. ${ }^{2}$ Extracellular BDNF appears to have two forms: pro-BDNF and mature $\mathrm{BDNF},{ }^{28}$ which are formed after cleavage of the precursor 
protein pre-pro-BDNF. ${ }^{8}$ Mature BDNF interacts with its high-affinity receptor, the tropomyosin-related kinase receptor type $B$ (TRKB), to exert its molecular effects in the adult CNS. ${ }^{2}$ BDNF induces dimerisation of TRKB with kinase activation and autophosphorylation of tyrosine residues, with subsequent activation of several adaptor proteins, which, in turn leads to phosphorylation of phosphoinositide 3-kinase, mitogen-activated protein kinase/ extracellular signal-regulated kinase, phospholipase $\mathrm{C} \gamma 1$ and cyclic adenosine monophosphate (cAMP)-responsive element-binding protein, all downstream pathways regulating neurite outgrowth, cell survival, cellular differentiation and synaptic plasticity. ${ }^{210}$ There is evidence that the cleaved prodomain of BDNF might be, by itself, an active biological modulator. ${ }^{11} 12$ BDNF and its cleaved propeptide have been found in large vesicles located in the presynaptic terminals of excitatory neurons in the adult hippocampus of mice. ${ }^{11}$ Interestingly, the Val66Met polymorphism of BDNF, which is located in the prodomain genomic region, alters substantially the prodomain structure. $^{12}$ Specifically, Met66 BDNF prodomain induces modulation of neuronal morphology through acute cone retraction. ${ }^{12}$

It is known that BDNF freely crosses the blood-brain barrier. ${ }^{13}$ In keeping with this observation, levels of BDNF in serum are correlated with CNS concentrations. ${ }^{14}$ Altered peripheral serum levels of BDNF have been shown to be a reliable biomarker for severe psychiatric disorders, including schizophrenia (SCZ). ${ }^{15}$ This corresponds to the dysregulation of BDNF and of its related molecular pathways observed in affected individuals. ${ }^{16}$

A decline in serum BDNF levels has been consistently identified in chronic and medicated SCZ patients, ${ }^{17-22}$ as well as in first-episode and medication-naive SCZ patients, ${ }^{23-27}$ compared with unaffected subjects. Conversely, other studies showed increased BDNF serum levels in patients with SCZ, ${ }^{28}{ }^{29}$ or have not been able to demonstrate differences in serum BDNF concentrations between drug-free or drug-naive patients and healthy controls. ${ }^{31}$ This discrepancy may depend on the clinical heterogeneity of the samples studied (ie, different stage of illness, subtype of SCZ and effect of medication), as well as on the different assessment tools employed. However, a recent quantitative meta-analytical estimate by Fernandes $e t a l^{32}$ confirmed that SCZ is associated with a moderate decrease of serum and plasma BDNF levels compared with healthy controls. This entails that decreased serum BDNF levels might be a marker of illness status in SCZ (ie, disease biomarker). There is a lack of knowledge, however, on the temporal manifestation of this decline. The decrease of peripheral BDNF could be constant, with premorbid levels roughly similar to those detected in unaffected individuals, linearly declining during the course of SCZ. Alternatively, BDNF peripheral levels might fluctuate in association with acute psychopathological phases of the disorder. On top of this, BDNF peripheral levels might vary as a result of drug treatments typically used in major psychoses, such as antipsychotics.
To date, however, the study of the relationship between BDNF serum levels and drug treatment in patients with SCZ and schizoaffective disorder (SAD) remains inconclusive. There is extensive preclinical evidence suggesting that typical antipsychotics might decrease BDNF expression whereas atypical antipsychotics, with the exception of risperidone, could increase BDNF expression. ${ }^{33}$ Clozapine-treated patients with chronic SCZ have higher BDNF levels than patients treated with typical neuroleptics ${ }^{1820}$ or with risperidone. ${ }^{18}$ Other studies, however, found no effect of antipsychotic treatment on the levels of serum BDNF. ${ }^{25} 3435$ Taken together, these data appear contradictory, making difficult to infer conclusions about the effects of antipsychotic treatment on serum BDNF levels. Meta-analytical findings, however, point to a small but significant increase of serum BDNF levels under antipsychotic treatment, although a class-specific effect has not been investigated. ${ }^{32}$

One final introductory remark concerns the link between BDNF and cognitive function in SCZ. Indeed, cognitive disruption seems to represent the core symptomatology that distinguishes patients with SCZ from those affected by other psychoses. Cognitive deficits are related to social integration, to social problem solving and to the acquisition of various skills ${ }^{36}$ and are considered the most powerful predictors of functional outcome in patients with SCZ. ${ }^{37}$ Cognitively impaired patients with chronic SCZ appear to show low-serum BDNF levels. ${ }^{38}$ A recent meta-analysis confirmed this observation, ${ }^{39}$ showing that higher levels of BDNF peripheral expression correlated with better cognitive performances in reasoning and problem-solving areas.

The apparent gap in the knowledge about the longitudinal variation of BDNF serum levels, and its relationship with clinical and treatment variables in major psychoses, needs to be filled by additional clinical research. Here, we present the design and methodology of a prospective observational study that takes advantage of a well-characterised population of patients with psychosis carefully monitored at an academic community health centre.

\section{Aims of the current study}

Our study has the following primary and secondary aims:

Primary aims:

1. Explore the longitudinal relationship between peripheral serum BDNF levels and variation in psychopathology in a cohort of patients with major psychosis recruited at different stages of the illness.

2. Assess whether variation of cognitive function correlates with peripheral BDNF serum levels.

3. Establish whether drug treatment impacts on the relationship between psychopathology and cognition with BDNF serum levels.

Secondary aims:

1. Study whether patients' baseline characteristics prior to enrolment, namely duration of illness, duration of untreated psychosis (DUP), psychiatric and somatic comorbidity, age at onset, gender, 


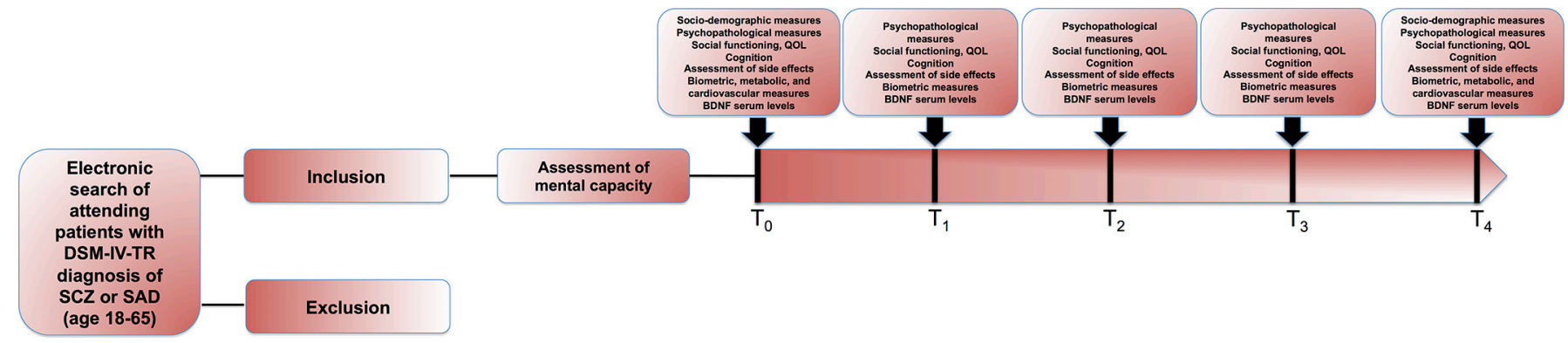

Figure 1 Flowchart of recruitment and assessment procedures for the Longitudinal assessment of BDNF in Sardinian psychotic patients study. BDNF, brain-derived neurotrophic factor; DSM-IV-TR, Diagnostic and Statistical Manual of Mental Disorders, Fourth Edition, Text Revision; QOL, quality of life; SAD, schizoaffective disorder; SCZ, schizophrenia.

family history of psychiatric disorders, premorbid adjustment and the type of illness course, might moderate the longitudinal relationship between peripheral serum BDNF and psychopathology.

2. Study whether measures of peripheral serum BDNF levels correlate with baseline measure of social functioning.

3. Analyse the relationship of peripheral BDNF levels with tolerability of antipsychotic treatment, as expressed by the presence of extrapyramidal symptoms (EPS).

\section{METHODS}

\section{Study design}

Longitudinal assessment of BDNF in Sardinian psychotic patients (LABSP) is a 24-month observational prospective cohort study. The sample will be recruited from patients followed up and treated at the community mental health centre of the Psychiatry Research Unit of the Department of Medical Science and Public Health, University of Cagliari and University of Cagliari Health Agency, Cagliari, Italy. Blood samples from recruited patients will be taken at baseline $\left(\mathrm{T}_{0}\right)$ and at three consecutive time points: 6 months $\left(\mathrm{T}_{1}\right), 12$ months $\left(\mathrm{T}_{2}\right)$ and 18 months $\left(\mathrm{T}_{3}\right)$ (figure 1). The temporal fluctuations of psychopathology, as well as the assessment of pharmacotherapy, measures of cognitive function and manifestation of treatment-related side effects (ie, EPS), will be also evaluated at each time point (table 1). The observational prospective design permits to disentangle how each variable might influence the outcome under study. Thus, we will assess whether changes in peripheral serum levels of BDNF predate or, alternatively, are consequent to psychopathological changes and/or drug treatment. As we will study the trajectory of peripheral serum BDNF levels over time, the recruited cohort will serve as its own control. We also expect to obtain an accurate estimation of the patterns of association between BDNF serum levels and all the independent clinical variables under consideration.

\section{Participants and recruitment}

The recruitment of the sample will be based on a two-step process. In the first step, all patients followed up at the community mental health centre of the University of Cagliari Health Agency, Cagliari, Italy with a diagnosis of SCZ or SAD according to the Diagnostic and Statistical Manual of Mental Disorders-IV-Text Revision $(\text { DSM-IV-TR })^{40}$ will be identified in the medical record database. The electronic search will be performed using lower (18) and upper (65) age limits, with no constraint regarding the presence of comorbid psychiatric disorders. After eligible patients will be identified in the database, the diagnosis of SCZ or SAD will be confirmed using the Structured Clinical Interview for DSM-IV-TR Axis I Disorders, Patient Edition (SCID-I/P) ${ }^{41}$ administered by trained mental health professionals (psychiatry residents or senior clinical staff). Further, we will collect detailed information on pharmacological treatment regime. Given the characteristics of the patient population followed up at our community mental health centre, we anticipate that our sample will not be comprised of drug-naive patients and will be on a pharmacological treatment regime mainly based on antipsychotics.

\section{Inclusion and exclusion criteria}

Inclusion criteria are: (1) age between 18 and 65 years; (2) diagnosis of SCZ or SAD according to DSM-IV-TR and (3) stability during the 6 months before recruitment. Exclusion criteria are: (1) refusal to provide consent; (2) presence of acute psychopathological symptoms; (3) presence of illness-related cognitive impairment of such severity that affects their ability to cooperate; (4) presence of major unstable medical illness; (5) severe mental retardation; (6) major neurological disorder or previous head injury; (7) current drug and alcohol dependence.

\section{Sample size and power calculation}

Our main null hypotheses are that there is no difference between mean BDNF serum levels at different time points in relation to: (1) the longitudinal psychopathological changes of patients with SCZ and SAD; (2) measures of cognitive functioning and (3) drug treatment. We based our power analysis on previous findings of BDNF serum levels longitudinal variation in a small cohort $(n=21)$ of patients with first-episode SCZ. ${ }^{24}$ Specifically, Palomino et al performed a 1-year prospective assessment of BDNF finding an absolute mean difference between baseline and the last time point of $1.65 .{ }^{24}$ Thus, a sample size of 59 
Open Access

Table 1 Assessment protocol in the LABSP

\section{Time points}

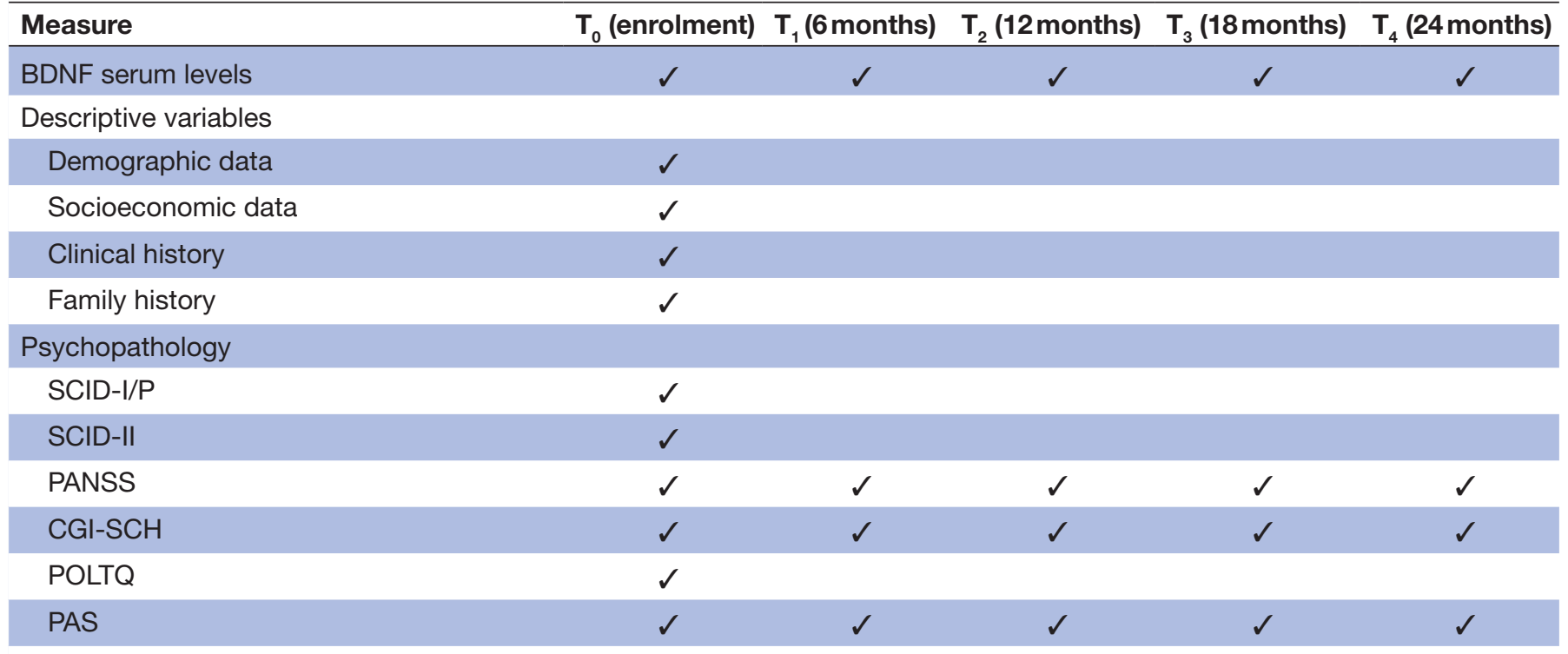

Social functioning and quality of life

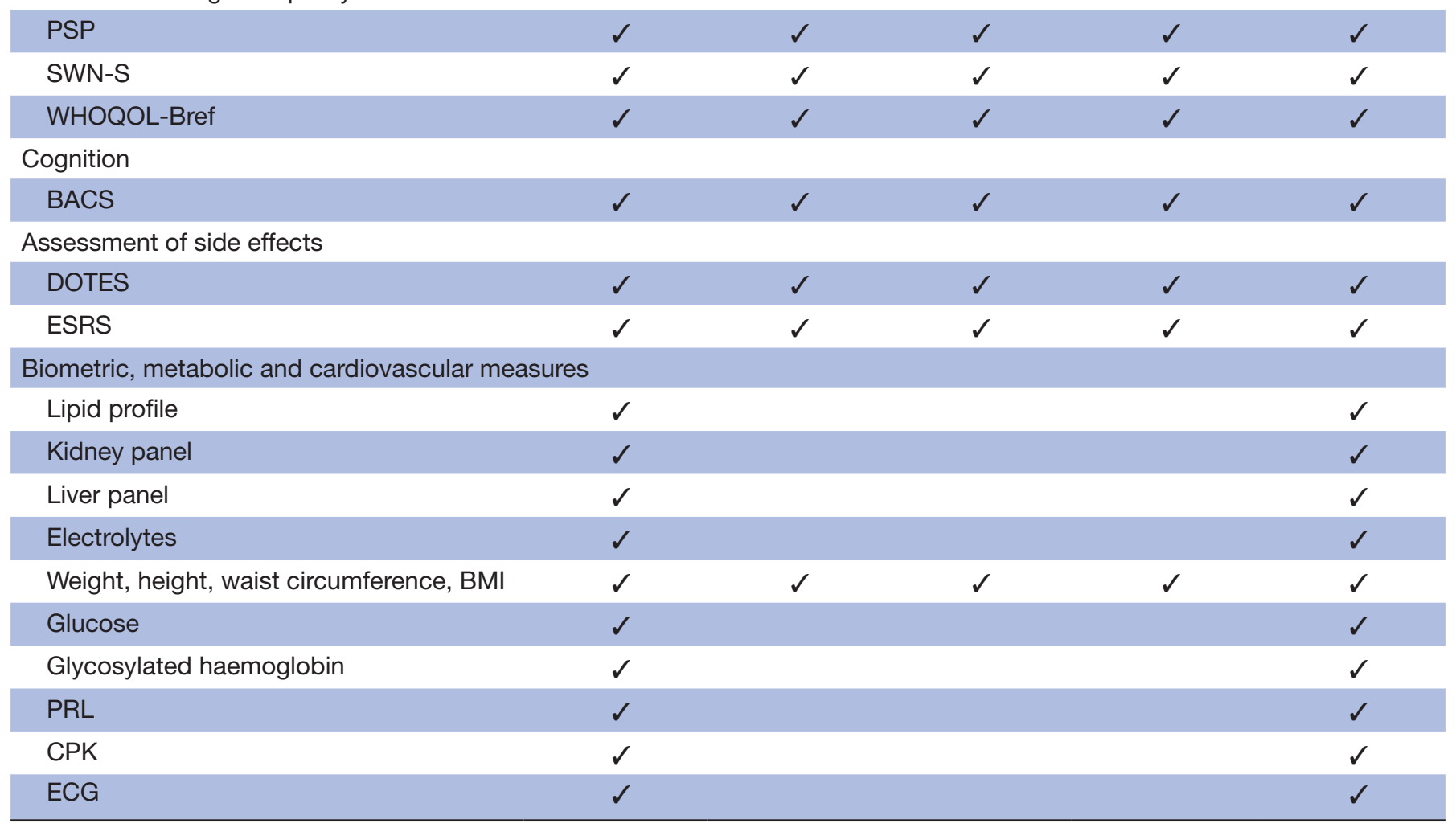

BACS, Brief Assessment of Cognition in Schizophrenia; BDNF, brain-derived neurotrophic factor; BMI, body mass index; CGI-SCH, Clinical Global Impression Scale for Schizophrenia; CPK, creatine phosphokinase; DOTES, Dosage Record Treatment Emergent Symptom Scale; ESRS, Extrapyramidal Symptom Rating Scale; LABSP, Longitudinal assessment of BDNF in Sardinian psychotic patients; PANSS, Positive and Negative Syndrome Scale; PAS, Premorbid Adjustment Scale; POLTQ, Psychopathological Onset Latency and Treatment Questionnaire; PRL, prolactin; PSP, Personal and Social Performance Scale; SCID-II, Structured Clinical Interview for DSM IV Axis II Disorders; SCID-I/P, Structured Clinical Interview for DSM IV Axis I Disorders; SWN-S, Subjective Well-Being under Neuroleptics-Short Version; WHOQOL-Bref, WHO Quality of Life-Bref.

individuals would be sufficient to obtain a $90 \%$ statistical power to detect significant difference at $\alpha=0.05$ between mean serum BDNF levels of this magnitude at each time point, considering an attrition rate for time point of
$5 \%$. Power analysis has been performed using repeated measures and sample size (RMASS) software. ${ }^{42}$ 


\section{Measures and materials}

Sociodemographic variables, personal and family history data Sociodemographic data will be collected using the Association for Methodology and Documentation in Psychiatry $(\mathrm{AMDP})^{43}$ assessment tool. Briefly, we will gather information on gender, age, level of education, marital status, offspring, economic status and occupation. Further, we will collect data on previous and current substance and/ or alcohol abuse and smoking status. The latter will be quantified also as number of cigarettes smoked per day. Information on family history of any psychiatric disorders will be collected through direct interview of the participant and at least one first-degree relative or significant other, as well as through an accurate review of available medical records. We will gather information on the stage of menstrual cycle whenever available. Finally, the systematic review of clinical records will allow us to retrospectively collect data on the presence of a previous history of stressful life events (SLE), including childhood maltreatment. We will also monitor longitudinally the eventual manifestation of SLE.

\section{Psychopathological measures}

The main psychopathological measures will be collected through the AMDP syndrome scale, ${ }^{43}$ which has shown to be valid in discriminating various forms of psychoses, as well as in providing a detailed psychopathological description of the subjects under study. ${ }^{44}$ As previously reported, patients' diagnosis will be confirmed using the Structured Clinical Interview (SCID)-I/P. ${ }^{41}$ Furthermore, all recruited patients will undergo assessment with the SCID for DSM-IV Axis II Personality Disorders (SCID-II) ${ }^{45}$ to detect the presence of comorbid personality disorders. Information on age of onset, age at first intervention (pharmacological and/or psychotherapeutic and/or psychosocial) will be based on retrospective information collected through direct interview of the patient, and at least one relative or significant other, as well as through an accurate review of available medical records. This approach should minimise the impact of recall bias on the assessment of key clinical measures.

We will also estimate DUP, the interval between the age of onset of full-blown psychotic symptoms and first antipsychotic treatment, using the Psychopathological Onset Latency and Treatment Questionnaire. ${ }^{46}$ Indeed a longer DUP appears to be related to a worse short-term ${ }^{47}$ and long-term clinical outcome ${ }^{48} 49$ in SCZ individuals, as well as with lower BDNF serum levels, ${ }^{50}$ although the latter finding has been not replicated in subsequent studies. ${ }^{51}$ Other measures of illness severity, such as number of hospital admissions and type of clinical course will be collected for the analysis.

Psychopathological assessment will include the Positive and Negative Symptom Scale for schizophrenia ${ }^{52}$ and the Clinical Global Impression Scale for Schizophrenia. ${ }^{53}$ The latter measure is a valid assessment tool for monitoring longitudinal psychopathological symptoms in routine clinical practice. ${ }^{54}$ Moreover, we will assess premorbid dysfunction, a well established prognostic marker in patients with SCZ, using the Premorbid Adjustment Scale. ${ }^{55}$ All these psychopathological measures will be analysed in relation to BDNF serum levels.

\section{Social functioning and quality of life measures}

Evaluation of social functioning will be carried out with the Personal and Social Performance Scale ${ }^{56}$ which showed good reliability in patients with SCZ. ${ }^{57}$ Further, assessment of quality of life (QOL) will be performed using the WHO Quality of Life-BREF (WHOQOL) version which has shown good validity and reliability in variety of psychiatric illnesses including SCZ.$^{58}{ }^{59}$ Finally, we will collect data using the Subjective Well-Being under Neuroleptics-Short Version. ${ }^{60}$

\section{Cognitive measures}

The cognitive performance will be examined in a standardised way using the Brief Assessment of Cognition in Schizophrenia Scale (BACS) ${ }^{61}$ It takes approximately half an hour to administer in healthy controls and includes brief assessments of four of the seven neurocognitive domains designated as important by the Measurement and Treatment Research to Improve Cognition in Schizophrenia battery: reasoning and problem solving, processing speed, verbal memory and working memory. The composite score has been shown to have high test-retest reliability in patients with SCZ. ${ }^{61}$ Moreover, the BACS appears to assess reliable measures of functional capacity. ${ }^{62}$ The BACS will permit a rapid assessment of cognitive function at different time points, allowing correlation of these measures with peripheral serum BDNF levels. Indeed, several studies have shown that peripheral serum BDNF level might be a biomarker of cognitive function in SCZ. ${ }^{63-66}$

\section{Assessment of side effects}

We will monitor the onset of antipsychotics-related side effects using the Dosage Record Treatment Emergent Symptoms Scale $^{67}$ and the Extrapyramidal Symptom Rating Scale. ${ }^{68}$ Of note, one study showed that patients with SCZ with tardive dyskinesia (TD) had lower plasma BDNF levels than those without TD. ${ }^{69}$ Data on antipsychotics dosages will be collected and converted to chlorpromazine equivalents for data analysis.

\section{Biometric, metabolic and cardiovascular measures}

BDNF appears to regulate appetite and weight regulation. Indeed, BDNF dysregulation has been detected in eating disorders, and might be determined via modulation of the serotonin system. In fact, infusion of BDNF in the hypothalamus of rats has been shown to increase serotonin turnover and suppress appetite. ${ }^{70}$ Further support for the regulatory role of BDNF in appetite and weight through modulation of the serotonin system comes from studies showing that mice with only one copy of the BDNF gene displayed increased food intake and weight. ${ }^{71}$ This corresponded to a decreased serotonin metabolite to serotonin ratio. Further, deletion of the BDNF gene 
leads to similar hyperphagic and obese phenotypes. ${ }^{72}$ There is also evidence suggesting that BDNF may regulate weight and food intake through the dopamine system. ${ }^{73} 74$ In addition, the attentional bias to food cues in low-restrained obese or overweight individuals was significantly altered by antagonism on dopamine receptors $3 .^{75}$ Based on these findings, we will evaluate the following clinical parameters: weight, waist circumference, body mass index, liver and kidney function panel, serum glucose, glycosylated haemoglobin, lipid profile, prolactin and creatine phosphokinase. The cardiovascular function will be monitored by ECG, with evaluation of QTc interval. Finally, we will collect information on exercise as this can impact on BDNF levels. ${ }^{76}$

\section{Sampling and assessment of BDNF serum levels}

Blood samples for each patient will be taken at the same time of the day (between 8:00 and 10:00 AM). BDNF serum levels will be evaluated using BDNF ELISA Kit (Booster Immunoleader Biological Technology Co., Ltd., Pleasanton, CA, USA, catalogue no EK0307) for the quantitative detection of human BDNF in cell culture supernatants, serum and plasma. This kit is based on a standard sandwich ELISA technology for specific quantifications of natural and recombinant human BDNF with a high sensitivity $(<2 \mathrm{pg} / \mathrm{mL})$ and with no detectable cross-reactivity with other relevant proteins. After blood sampling, serum will be allowed to clot in a serum separator tube for about 4 hours at room temperature. After that, it will be centrifuged at approximately $1000 \times \mathrm{g}$ for $15 \mathrm{~min}$. Supernatant serum samples will be collected in small aliquot and stored immediately at $-20^{\circ} \mathrm{C}$ for future analysis. Then, samples will be processed according to kit protocol and instructions. Optical density absorbance of each sample will be read with a $450 \mathrm{~nm}$ filter in a microplate reader (Thermo Scientific Multiskan FC, Thermo Fisher Scientific Oy Ratastie 2, Finland) within 30 min after the final step of the kit procedure. Data obtained will be analysed using the Thermo Scientific SkanIt Software V.3.0 for Multiskan FC.

\section{Data analysis plan}

Mixed-effects linear regression models (MLRM) will be used to analyse longitudinal data. ${ }^{77} 78$ Specifically, we will regress independent variables (both categorical and continuous) on BDNF serum levels (dependent variable). MLRM allow to model individual change over time and appear to be more flexible in terms of repeated measures, particularly when the number of observations per subject is not the same at each time point. ${ }^{77} 78$ Further, these models allow generalisation of non-normally distributed data for independent variables. Our analysis plan will consist of the following steps. First, we will perform a visual inspection of mean BDNF serum levels at each time point using boxplots. This will allow checking for normality of BDNF serum levels at each time point as well as to identify outliers. Second, MLRM will be analysed to assess the longitudinal variation of BDNF levels while correcting for age and sex. Finally, independent variables will be regressed on BDNF serum levels. Covariates will be added to significant models to account for possible intercorrelations. All data will be analysed using 'Ime4' package implemented in R. ${ }^{79}$ Missing data for independent variables will be dealt with the "na. action' function implemented in R. The statistical significance of identified MLRM will be calculated using the 'multcomp' R package. Finally, graphical representation of MLRM will be obtained with R packages 'sJPlot' and 'sjmisc'.

\section{Ethics and dissemination}

This study protocol was approved by the University of Cagliari Health Agency Ethics Committee (NP2016/5491). The study will be conducted in accordance with the principles of good clinical practice in the Declaration of Helsinki in compliance with the regulations. Written informed consent will be obtained from all eligible participants. Participation to the study will be voluntary and patients will be able to withdraw consent at any point with no disadvantage to their treatments. A psychiatric assessment will establish that patients' ability to consent is not compromised by their psychopathological status.

We plan to disseminate the results of our study through conference presentations and publication in international peer-reviewed journals. Access to raw data will be available in anonymised form upon request to the corresponding author.

\section{DISCUSSION}

Schizophreniais characterised by three distinct, but to a certain extent intertwined, clusters of symptoms: positive, negative and cognitive. Each one of these clusters presents with a varying degree of severity and with a specific prevalence depending on the stage of the illness. ${ }^{80}$ Patients with stable diagnoses of SCZ and SAD have often incomplete remissions (particularly from negative symptoms), and not rarely recurrences or relapses of positive symptoms, mainly delusions and hallucinations. ${ }^{81}$ Cognitive function, however, appears to decline steadily over the course of the illness, although most of the magnitude of the differences in cognition between patients with SCZ and unaffected subjects appear to be modulated by age. ${ }^{81}$

One key aspect in patients with diagnostically stable SCZ and SAD is being able to predict the manifestation of these psychopathological exacerbations. Certainly, given that fluctuations appear to be more prominent for positive symptoms and to a lesser extent for negative ones, rather than for cognition, researchers have focused on the identification of prodromal signs of relapses/recurrences using, for instance, home telemonitoring of symptoms change via mobile phone-based platforms. ${ }^{82} 83$ This approach has proven effective in reducing the number 
of relapses and consequent hospitalisations in individuals with psychosis. ${ }^{82} 83$

Our prospective study, LABSP, expands on this evidence aiming at monitoring not only the clinical signs of patients with SCZ over a 24-month period, but, more importantly, assessing the predictive power of a well-established biomarker of illness status in SCZ (BDNF serum levels), on the clinical trajectory of SCZ. We expect to find correlation of BDNF levels with longitudinal variation of psychopathology, particularly concerning positive symptoms. This prospective approach, to our knowledge, has not been proposed previously in the literature, and might offer novel data on serum BDNF as a biomarker of psychopathological changes in accurately monitored patients with SCZ. If such a correlation will be identified, the joint analysis of clinical and BDNF data might explain a larger proportion of psychopathological change than clinical data alone.

One additional point of novelty concerns the analysis of the impact of psychiatric comorbidity on BDNF levels. Indeed, SCZ is not rarely associated with other axis I and II disorders that typically affect negatively the course and outcome of the disorder. ${ }^{84}$ However, to our knowledge the relationship between serum BDNF levels in SCZ and psychiatric comorbidities has not been tested in previous work. Although this analysis remains a secondary aim of our study, the sample size should allow an adequately powered post hoc stratification of our prospective data to test this relationship.

The wealth of clinical data we are planning to collect with LABSP will also allow testing the moderating influence of relevant clinical variables, such as for instance, family history of SCZ. In fact, it is plausible that recruited participants with a positive family history of SCZ, will have lower baseline BDNF levels compared with those without a family history and a different pattern of association between serum BDNF levels and the various clinical independent variables.

\section{Limitations}

Our study has some limitations inherent to its design. First, we will study a population recruited in a tertiary clinic specialised in treatment of patients with psychosis, possibly limiting ecological validity (ie, generalisability) of the results. Second, our power analysis is based on previous longitudinal estimates of 1.65 mean variation of serum BDNF levels over a 1-year follow-up. Although a sample size of 59 might be sufficient to detect a longitudinal variation of this magnitude in BDNF serum levels we might not be able to detect association signals of small effect size between clinical, treatment and cognitive variables and BDNF. Thus, we plan to extend our recruitment well beyond the number provided by our power analysis. Finally, notwithstanding the prospective design, clinical (illness duration, DUP) and treatment history prior to study entry might impact on findings.

\section{CONCLUSIONS}

In summary, LABSP will allow the prospective assessment of BDNF serum levels, as well as of key clinical and treatment-related measures, in a relatively large cohort of patients with SCZ and SAD followed up in a naturalistic setting. This work will provide useful information on the relationship between BDNF serum levels and psychopathological changes over time. Changes in BDNF peripheral levels might be predictive of the psychopathological trajectory during the 24-month follow-up, and if validated in independent samples, might point to a role of BDNF as a biomarker of clinical trajectory in patients with psychosis.

Contributors DP contributed to the design of the study and drafted the first version of the manuscript. $\mathrm{BC}$ conceived the study, led the study team and critically revised the manuscript. MM contributed to the assessment protocol, to the design of the study and to the drafting of the manuscript. LD and MT contributed to the study design and assessments. MS and RC contributed to brain-derived neurotrophicfactor (BDNF) serum levels assessments and laboratory procedures. PF and WF designed the experimental procedures for BDNF assessment. All authors read and approved the final version of the manuscript.

Funding This study is funded through the grant 'Fondo integrativo per la ricerca' of the University of Cagliari (BC).

Competing interests None declared.

Patient consent Obtained.

Ethics approval This study protocol was approved by the University of Cagliari Health Agency Ethics Committee (NP2016/5491).

Provenance and peer review Not commissioned; externally peer reviewed.

Open Access This is an Open Access article distributed in accordance with the Creative Commons Attribution Non Commercial (CC BY-NC 4.0) license, which permits others to distribute, remix, adapt, build upon this work non-commercially, and license their derivative works on different terms, provided the original work is properly cited and the use is non-commercial. See: http://creativecommons.org/ licenses/by-nc/4.0/

(c) Article author(s) (or their employer(s) unless otherwise stated in the text of the article) 2017. All rights reserved. No commercial use is permitted unless otherwise expressly granted.

\section{REFERENCES}

1 Binder DK, Scharfman HE. Factor B-derived neurotrophic factor. Growth Factors 2004;22:123-31.

2. Nagahara AH, Tuszynski MH. Potential therapeutic uses of BDNF in neurological and psychiatric disorders. Nat Rev Drug Discov 2011;10:209-19.

3. Huang ZJ, Kirkwood A, Pizzorusso T, et al. BDNF regulates the maturation of inhibition and the critical period of plasticity in mouse visual cortex. Cell 1999;98:739-55.

4. Nawa H, Takahashi M, Patterson PH. Cytokine and growth factor involvement in schizophrenia--support for the developmental model. Mol Psychiatry 2000;5:594-603.

5. Gorski JA, Zeiler SR, Tamowski S, et al. Brain-derived neurotrophic factor is required for the maintenance of cortical dendrites. $J$ Neurosci 2003;23:6856-65.

6. Pang PT, Lu B. Regulation of late-phase LTP and long-term memory in normal and aging Hippocampus: role of secreted proteins IPA and BDNF. Ageing Res Rev 2004;3:407-30.

7. Barde YA, Edgar D, Thoenen H. Purification of a new neurotrophic factor from mammalian brain. Embo J 1982;1:549-53.

8. Autry AE, Monteggia LM. Brain-derived neurotrophic factor and neuropsychiatric disorders. Pharmacol Rev 2012;64:238-58.

9. Lessmann V, Gottmann K, Malcangio M. Neurotrophin secretion: current facts and future prospects. Prog Neurobiol 2003;69:341-74

10. Huang EJ, Reichardt LF. Trk receptors: roles in neuronal signal transduction. Annu Rev Biochem 2003;72:609-42. 
11. Dieni S, Matsumoto T, Dekkers M, et al. BDNF and its pro-peptide are stored in presynaptic dense core vesicles in brain neurons. J Cell Biol 2012;196:775-88.

12. Anastasia A, Deinhardt K, Chao MV, et al. Val66Met polymorphism of BDNF alters prodomain structure to induce neuronal growth cone retraction. Nat Commun 2013;4:2490.

13. Pan W, Banks WA, Fasold MB, et al. Transport of brainderived neurotrophic factor across the blood-brain barrier. Neuropharmacology 1998;37:1553-61.

14. Klein AB, Williamson R, Santini MA, et al. Blood BDNF concentrations reflect brain-tissue BDNF levels across species. Int $J$ Neuropsychopharmacol 2011:14:347-53

15. Fernandes BS, Berk M, Turck CW, et al. Decreased peripheral brainderived neurotrophic factor levels are a biomarker of disease activity in major psychiatric disorders: a comparative meta-analysis. Mol Psychiatry 2014;19:750-1.

16. Angelucci $F$, Brenè $S$, Mathé AA. BDNF in schizophrenia, depression and corresponding animal models. Mol Psychiatry 2005;10:345-52.

17. Toyooka K, Asama K, Watanabe $\mathrm{Y}$, et al. Decreased levels of brainderived neurotrophic factor in serum of chronic schizophrenic patients. Psychiatry Res 2002;110:249-57.

18. Tan YL, Zhou DF, Cao LY, et al. Decreased BDNF in serum of patients with chronic schizophrenia on long-term treatment with antipsychotics. Neurosci Lett 2005;382:27-32.

19. Zhang XY, Tan YL, Zhou DF, et al. Serum BDNF levels and weight gain in schizophrenic patients on long-term treatment with antipsychotics. J Psychiatr Res 2007:41:997-1004.

20. Grillo RW, Ottoni GL, Leke R, et al. Reduced serum BDNF levels in schizophrenic patients on clozapine or typical antipsychotics. $J$ Psychiatr Res 2007;41:31-5.

21. Ikeda Y, Yahata N, Ito I, et al. Low serum levels of brain-derived neurotrophic factor and epidermal growth factor in patients with chronic schizophrenia. Schizophr Res 2008;101:58-66.

22. Xiu MH, Hui L, Dang YF, et al. Decreased serum BDNF levels in chronic institutionalized schizophrenia on long-term treatment with typical and atypical antipsychotics. Prog Neuropsychopharmacol Biol Psychiatry 2009;33:1508-12.

23. Buckley PF, Pillai A, Evans D, et al. Brain derived neurotropic factor in first-episode psychosis. Schizophr Res 2007;91:1-5.

24. Palomino A, Vallejo-Illarramendi A, González-Pinto A, et al. Decreased levels of plasma BDNF in first-episode schizophrenia and bipolar disorder patients. Schizophr Res 2006;86:321-2.

25. Pirildar S, Gönül AS, Taneli F, et al. Low serum levels of brain-derived neurotrophic factor in patients with schizophrenia do not elevate after antipsychotic treatment. Prog Neuropsychopharmacol Biol Psychiatry 2004;28:709-13.

26. Rizos EN, Rontos I, Laskos E, et al. Investigation of serum BDNF levels in drug-naive patients with schizophrenia. Prog Neuropsychopharmacol Biol Psychiatry 2008;32:1308-11.

27. Chen DC, Wang J, Wang B, dC C, Wang B, et al. Decreased levels of serum brain-derived neurotrophic factor in drug-naïve first-episode schizophrenia: relationship to clinical phenotypes. Psychopharmacology 2009;207:375-80.

28. Gama CS, Andreazza AC, Kunz M, et al. Serum levels of brainderived neurotrophic factor in patients with schizophrenia and bipolar disorder. Neurosci Lett 2007;420:45-8.

29. Reis HJ, Nicolato R, Barbosa IG, et al. Increased serum levels of brain-derived neurotrophic factor in chronic institutionalized patients with schizophrenia. Neurosci Lett 2008:439:157-9.

30. Huang TL, Lee CT. Associations between serum brain-derived neurotrophic factor levels and clinical phenotypes in schizophrenia patients. J Psychiatr Res 2006;40:664-8.

31. Shimizu E, Hashimoto K, Watanabe H, et al. Serum brainderived neurotrophic factor (BDNF) levels in schizophrenia are indistinguishable from controls. Neurosci Lett 2003;351:111-4.

32. Fernandes BS, Steiner J, Berk M, et al. Peripheral brain-derived neurotrophic factor in schizophrenia and the role of antipsychotics: meta-analysis and implications. Mol Psychiatry 2015;20:1108-19.

33. Favalli G, Li J, Belmonte-de-Abreu P, et al. The role of BDNF in the pathophysiology and treatment of schizophrenia. J Psychiatr Res 2012;46:1-11.

34. Yoshimura R, Hori H, Sugita A, et al. Treatment with risperidone for 4 weeks increased plasma 3-methoxy-4-hydroxypnenylglycol (MHPG) levels, but did not alter plasma brain-derived neurotrophic factor (BDNF) levels in schizophrenic patients. Prog Neuropsychopharmacol Biol Psychiatry 2007;31:1072-7.

35. Hori $\mathrm{H}$, Yoshimura R, Yamada Y, et al. Effects of olanzapine on plasma levels of catecholamine metabolites, cytokines, and brainderived neurotrophic factor in schizophrenic patients. Int Clin Psychopharmacol 2007;22:21-7.
36. Green MF. What are the functional consequences of neurocognitive deficits in schizophrenia? Am J Psychiatry 1996;153:321-30.

37. Green MF, Kern RS, Heaton RK. Longitudinal studies of cognition and functional outcome in schizophrenia: implications for MATRICS. Schizophr Res 2004;72:41-51.

38. Zhang XY, Chen DC, Xiu MH, et al. Gender differences in nevermedicated first-episode schizophrenia and medicated chronic schizophrenia patients. J Clin Psychiatry 2012;73:1025-33.

39. Ahmed AO, Mantini AM, Fridberg DJ, et al. BDNF) and neurocognitive deficits in people with schizophrenia: a meta-analysis. Psychiatry Res 2015;226:1-13.

40. American Psychiatric Association. American Psychiatric Association: Diagnostic and Statistical Manual of Mental Disorders. 4th ed. Washington, DC: American Psychiatri Association, 2000.

41. First MB, Spitzer RL, Gibbon M, et al. Structured clinical interview for DSM-IV-TR Axis I disorders, research version, patient edition. (SCIDI/P). New York: Biometrics Research, New York State Psychiatric Institute, 2002.

42. Roy A, Bhaumik DK, Aryal S, et al. Sample size determination for hierarchical longitudinal designs with differential attrition rates. Biometrics 2007;63:699-707.

43. Conti L, Dell'Osso L, Cassano GB. II sistema AMDP. Manuale per la valutazione e la documentazione della psicopatologia. Milano: Edizioni Grafiche Mazzucchelli, 1990.

44. Gebhardt R, Pietzcker A. (Validity of the syndrome scales in the AMDP-system). Arch Psychiatr Nervenkr 19701983;233:509-23;233:509-23.

45. First MB, Gibbon M, Spitzer RL, et al; Structured clinical interview for DSM-IV Axis II Personality Disorders, (SCID-II. Washington, D.C.: American Psychiatric Press, Inc, 1997.

46. Dell'Osso B, Palazzo M, Primavera D, et al. P-597 - Determination of psychopatological onset and latency to treatment in psychiatric disorders through the 'psychopatological onset and latency to treatment questionnaire'. European Psychiatry 2012;27:1.

47. Perkins DO, Gu H, Boteva K, et al. Relationship between duration of untreated psychosis and outcome in first-episode schizophrenia: a critical review and meta-analysis. Am J Psychiatry 2005;162:1785-804.

48. Primavera D, Bandecchi C, Lepori T, et al. Does duration of untreated psychosis predict very long term outcome of schizophrenic disorders? results of a retrospective study. Ann Gen Psychiatry 2012;11:21

49. Penttilä M, Jääskeläinen $\mathrm{E}$, Hirvonen $\mathrm{N}$, et al. Duration of untreated psychosis as predictor of long-term outcome in schizophrenia: systematic review and meta-analysis. $\mathrm{Br} J$ Psychiatry 2014:205:88-94

50. Rizos EN, Michalopoulou PG, Siafakas N, et al. Association of serum brain-derived neurotrophic factor and duration of untreated psychosis in first-episode patients with schizophrenia. Neuropsychobiology 2010;62:87-90.

51. Şimşek S, Gençoğlan S, Yüksel T, et al. Lower brain-derived neurotropic factor levels in untreated adolescents with first-episode psychosis. J Clin Psychopharmacol 2015;35:596-9.

52. Kay SR, Fiszbein A, Opler LA. The positive and negative syndrome scale (PANSS) for schizophrenia. Schizophr Bull 1987;13:261-76.

53. Haro JM, Kamath SA, Ochoa S, et al. The Clinical Global ImpressionSchizophrenia scale: a simple instrument to measure the diversity of symptoms present in schizophrenia. Acta Psychiatr Scand Suppl 2003:16-23.

54. Pinna F, Deriu L, Diana E, et al. Clinical Global Impression-severity score as a reliable measure for routine evaluation of remission in schizophrenia and schizoaffective disorders. Ann Gen Psychiatry 2015:14:6.

55. Cannon-Spoor HE, Potkin SG, Wyatt RJ. Measurement of premorbid adjustment in chronic schizophrenia. Schizophr Bull 1982;8:470-84.

56. Goldman HH, Skodol AE, Lave TR. Revising axis V for DSMIV: a review of measures of social functioning. Am J Psychiatry 1992;149:1148-56.

57. Nasrallah H, Morosini P, Gagnon DD, Reliability GDD. Reliability, validity and ability to detect change of the Personal and Social Performance scale in patients with stable schizophrenia. Psychiatry Res 2008;161:213-24.

58. Skevington SM, Lotfy M, O'Connell KA. WHOQOL Group. The World Health Organization's WHOQOL-BREF quality of life assessment: psychometric properties and results of the international field trial. A report from the WHOQOL group. Qual Life Res 2004;13:299-310.

59. Carpiniello B, Pinna M, Carta MG, et al. Reliability, validity and acceptability of the WHOQOL-Bref in a sample of Italian psychiatric outpatients. Epidemiol Psichiatr Soc 2006;15:228-32.

60. Naber D. A self-rating to measure subjective effects of neuroleptic drugs, relationships to objective psychopathology, quality of life, 
compliance and other clinical variables. Int Clin Psychopharmacol 1995;10(Suppl 3):133-8.

61. Keefe RS, Goldberg TE, Harvey PD, et al. The Brief Assessment of Cognition in Schizophrenia: reliability, sensitivity, and comparison with a standard neurocognitive battery. Schizophr Res 2004;68:283-97.

62. Keefe RS, Poe M, Walker TM, et al. The relationship of the Brief assessment of Cognition in Schizophrenia (BACS) to functional capacity and real-world functional outcome. $J$ Clin Exp Neuropsychol 2006;28:260-9.

63. Vinogradov S, Fisher M, Holland C, et al. Is serum brain-derived neurotrophic factor a biomarker for cognitive enhancement in schizophrenia? Biol Psychiatry 2009;66:549-53.

64. Nieto R, Kukuljan M, Silva H, Bdnf SH. BDNF and schizophrenia: from neurodevelopment to neuronal plasticity, learning, and memory. Front Psychiatry 2013;4:45.

65. Goff DC. Future perspectives on the treatment of cognitive deficits and negative symptoms in schizophrenia. World Psychiatry 2013;12:99-107.

66. Zhang $\mathrm{XY}$, Liang J, Chen DC, dC C, et al. Low BDNF is associated with cognitive impairment in chronic patients with schizophrenia. Psychopharmacology 2012;222:277-84.

67. Garvey CA, Gross D, Freeman L. Assessing psychotropic medication side effects among children. A reliability study. J Child Adolesc Psychiatr Ment Health Nurs 1991;4:127-31.

68. Chouinard G, Margolese HC. Manual for the Extrapyramidal Symptom Rating Scale (ESRS). Schizophr Res 2005;76:247-65

69. Tan YL, Zhou DF, Zhang XY. Decreased plasma brain-derived neurotrophic factor levels in schizophrenic patients with tardive dyskinesia: association with dyskinetic movements. Schizophr Res 2005;74:263-70.

70. Pelleymounter MA, Cullen MJ, Wellman CL. Characteristics of BDNFinduced weight loss. Exp Neurol 1995;131:229-38.

71. Lyons WE, Mamounas LA, Ricaurte GA, et al. Brain-derived neurotrophic factor-deficient mice develop aggressiveness and hyperphagia in conjunction with brain serotonergic abnormalities. Proc Natl Acad Sci USA 1999;96:15239-44.

72. Rios M, Fan G, Fekete C, et al. Conditional deletion of brain-derived neurotrophic factor in the postnatal brain leads to obesity and hyperactivity. Mol Endocrinol 2001;15:1748-57.
73. Geiger BM, Haburcak M, Avena NM, et al. Deficits of mesolimbic dopamine neurotransmission in rat dietary obesity. Neuroscience 2009;159:1193-9.

74. Johnson PM, Kenny PJ. Dopamine D2 receptors in addictionlike reward dysfunction and compulsive eating in obese rats. Nat Neurosci 2010;13:635-41.

75. Nathan PJ, O'Neill BV, Mogg K, et al. The effects of the dopamine $\mathrm{D} \square$ receptor antagonist GSK598809 on attentional Bias to palatable food cues in overweight and obese subjects. Int $J$ Neuropsychopharmacol 2012;15:149-61.

76. Szuhany KL, Bugatti M, Otto MW. A meta-analytic review of the effects of exercise on brain-derived neurotrophic factor. J Psychiatr Res 2015;60:56-64.

77. Hedeker D, Mermelstein RJ, Berbaum ML, et al. Modeling mood variation associated with smoking: an application of a heterogeneous mixed-effects model for analysis of ecological momentary assessment (EMA) data. Addiction 2009;104:297-307.

78. Hedeker D, Mermelstein RJ, Demirtas H. Modeling between-subject and within-subject variances in ecological momentary assessment data using mixed-effects location scale models. Stat Med 2012;31:3328-36.

79. Bates D, Maechler M, Bolker B, et al. Fitting linear mixed models using Ime4. J Stat Softw 2015;67:1-48.

80. Wood SJ, Yung AR, McGorry PD, et al. Neuroimaging and treatment evidence for clinical staging in psychotic disorders: from the at-risk mental state to chronic schizophrenia. Biol Psychiatry 2011;70:619-25.

81. Heilbronner U, Samara M, Leucht S, et al. The longitudinal course of schizophrenia across the lifespan: clinical, cognitive, and neurobiological aspects. Harv Rev Psychiatry 2016;24:118-28.

82. Spaniel F, Vohlídka P, Kozený J, et al. The Information Technology aided Relapse Prevention Programme in Schizophrenia: an extension of a mirror-design follow-up. Int J Clin Pract 2008:62:1943-6.

83. Spaniel F, Vohlídka P, Hrdlicka J, et al. ITAREPS: information technology aided relapse prevention programme in schizophrenia. Schizophr Res 2008;98:312-7.

84. Menezes NM, Arenovich T, Zipursky RB. A systematic review of longitudinal outcome studies of first-episode psychosis. Psychol Med 2006;36:1349-62. 